Bol. Acad. peru. leng. 66. 2019 (37-63)

\title{
VARIACIÓN Y CREACIÓN LÉXICA: ESTUDIO SOCIOLINGÜÍSTICO DE LA METÁTESIS EN LA CREACIÓN DE JERGAS DE LOS UNIVERSITARIOS LIMEÑOS
}

\section{VARIATION AND LEXICAL CREATION: SOCIOLINGUISTIC STUDY OF THE METÁTESIS IN THE CREATION OF JERGAS OF THE LIMEÑOS UNIVERSITARIES}

\author{
Claudia Giselle Nieto Salas \\ Juan Gerardo Paco Saavedra \\ Universidad Nacional Mayor de San Marcos
}

\section{Resumen:}

En el presente artículo damos cuenta de la variación lingüística dada por la creación de jergas mediante el proceso de metátesis. Para el presente estudio, la comunidad escogida fue la de los universitarios limeños. Dilucidamos cómo se presenta este proceso basado en las variables de edad, género, procedencia y facultad en la que estudian, las cuales son contrastadas para exponer de qué manera varía el uso del mismo de acuerdo con factores sociales. Finalmente, explicamos las razones de la utilización de este mecanismo en las relaciones de mayor familiaridad entre los hablantes.

\section{Abstract:}

In this article we give an accountof the linguistic variation made by the jargon creation through metathesis. Likewise, the study was focused https://doi.org/10.46744/bapl.201902.002 
on the university students from Lima. We elucidate how this process is shown based on variables of age, genre, origin and the faculty where they study, factors thatare contrasted to show how the use of this process varies based on social agents. Finally, we explain the reasons for the utilization of this mechanism on more-familiar relationships between the speakers.

Palabras clave: variación lingüística; jerga; metátesis; universitarios limeños.

Key words: linguistic variation; jargon; metathesis; university students.

Fecha de recepción:

$15 / 09 / 2019$

Fecha de aceptación:

$30 / 11 / 2019$

\section{Introducción}

El uso y la creación de jergas en la sociedad, sobre todo entre los hablantes jóvenes, es un ejemplo de variación lingüística que se encuentra presente en las distintas comunidades de habla. Estas variaciones se muestran de distintas maneras y bajo numerosos mecanismos por los cuales las palabras sufren ligeros o contundentes cambios: uno de ellos, quizá de los más utilizados en el habla coloquial limeña, es el proceso de metátesis. Por otra parte, la creación y variación léxicas enriquecen de tal manera el vocabulario del hablante y fortalece los lazos de familiaridad en los grupos sociales, y permiten que sus miembros se identifiquen con uno de estos grupos y desarrollen un sentido de pertenencia que los una al mismo mediante el desarrollo lingüístico.

La creación de jergas mediante el mecanismo de metátesis es común en el habla coloquial limeña; sin embargo, este fenómeno 
https://doi.org/10.46744/bapl.201902.002

no ha sido tratado de manera detenida por los investigadores, sino que aparece incluido dentro de estudios generales sobre el uso de jergas como lo muestra Ramírez (1996), quien identifica el proceso de creación de palabras por metátesis dentro de un habla particular que denomina replana limeña, a la cual dedica no más que unos cuantos párrafos. En este mismo sentido, se encuentran incontables trabajos que abordan el léxico de diversos grupos sociales, en especial los que intentan documentar el habla delincuencial o del bajo mundo, en los que el proceso de metátesis, si es que aparece, ocupa pocas líneas.

Distinta suerte han tenido trabajos similares en otros países. En Argentina, por ejemplo, Sorbet (2014) describe a profundidad el proceso de creación de jergas por metátesis en el español bonaerense. Este autor describe el vesre porteño, una forma particular de habla de Buenos Aires, nacida en el seno de la delincuencia, como parte de un dialecto mayor denominado lunfardo. Así como el vesre en Argentina, en otras lenguas se crearon hablas particulares que empleaban este mismo procedimiento, siendo uno de los más estudiados el verlan, en Francia, descrito por Calvet (1994). Cabe aclarar que, entre el vesre argentino, el verlan francés y el back slang inglés existe una clara diferencia con respecto a las jergas limeñas creadas por metátesis. Esta diferencia es la extensión del vocabulario; en la jerga limeña, se encuentra un vocabulario reducido, lo que evidencia una menor identificación y, por ende, una menor explotación de este recurso.

Como hemos visto, existe un «vacío» en cuanto al tratamiento que se le da al proceso de metátesis como mecanismo de creación de jergas en Lima, pues, aunque algunas palabras se encuentren documentadas, no existe un análisis que denote a qué responde tal creatividad en la innovación léxica, y de qué manera intervienen en su uso las variables sociolingüísticas. Es en este sentido que nos planteamos dos objetivos: determinar de qué manera varía el uso de las jergas creadas por metátesis atendiendo a las variables de género, edad, procedencia y facultad, y explicar las motivaciones de la utilización de las jergas creadas por metátesis teniendo en cuenta las relaciones de pertenencia y familiaridad. 
En cuanto a la organización, el presente artículo se encuentra estructurado de la siguiente manera: en primer lugar, presentamos un marco teórico que define los conceptos fundamentales de la investigación desde una perspectiva sociolingüística y de variación léxica, a saber, las nociones de variación, variables sociales, pertenencia, jerga y la metátesis como un proceso de creación de palabras. A continuación, exponemos en el tercer apartado los datos más resaltantes extraídos del análisis realizado a partir de las encuestas que fueron aplicadas a los jóvenes universitarios de las distintas áreas de estudio (facultades). En el cuarto apartado, brindamos las conclusiones obtenidas tras la investigación, que se condicen con los objetivos propuestos en el párrafo anterior. Finalmente, presentamos a modo de anexo el formato de la encuesta realizada a los universitarios que accedieron a participar del estudio y las definiciones de las jergas utilizadas en el estudio, culminando el presente artículo con la lista de referencias bibliográficas.

\section{Marco teórico}

\subsection{Variación lingüística}

La variación lingüística es un hecho que ha sido confirmado gracias al estudio sociolingüístico. Sabemos, como explicita Labov, que no existe hablante alguno que utilice un único estilo o registro, dado que cada uno de ellos manifiesta una variación específica de acuerdo con el contexto sociocultural en el cual se encuentra (Etxebarria, 2013). Así, encontramos distintos tipos de variación lingüística, siendo la léxica aquella de la que nos encargaremos en el presente artículo.

Durante el estudio de la variación léxica, la sociolingüística se encargará de "analizar las implicaciones del uso dentro de contextos discursivos, dialógicos y/o socioculturales» (Areiza et al., 2012: 25), y no solo de dar cuenta de las unidades léxicas aisladas. Así, Talmy (1985), de acuerdo con sus patrones de lexicalización, agrega que todo lo que es semántica y pragmáticamente importante en una determinada comunidad lingüística se codifica 
https://doi.org/10.46744/bapl.201902.002

gramaticalmente de forma explícita. Conociendo esta variación se podrá seleccionar un grupo dialectal de estudio (diafásico, en cuanto a lo que nos concierne) y apoyarse, a su vez, en el registro lingüístico utilizado por los hablantes en contextos específicos de la situación comunicativa.

Debido a que el estudio sociolingüístico pretende dar cuenta de «las variaciones lingüísticas dadas en el uso, en su relación con los factores sociales que las determinan y el papel que dichas variaciones desempeñan en el cambio lingüístico» (Areiza et al., 2012: 5), es importante realizar la investigación basada en las variedades sociales como factores que permiten el cambio lingüístico. Para lo que nos concierne en el presente estudio, nos limitaremos a abordar las variables de género y edad, siendo esta última la que nos encaminará directamente hacia nuestros objetivos al contrastar los resultados con las variables de procedencia y, en cierta medida, de profesión.

El género es una variable discutible, pero a la vez importante en el estudio sociolingüístico. Diversos autores indican que, por un lado, las mujeres son educadas para usar la lengua de forma cooperativa, con un lenguaje más cuidadoso y menos coloquial que los varones, quienes organizan sus actividades lúdicas marcando una jerarquía para preservar su estatus y prestigio personal. En torno a ello, Wardhaugh (2015) menciona: «one generalization that has been made is that changes toward more vernacular forms tend to be led by men, while changes toward the standard tend to be led by women» (Romaine, 2003: 208).

Por su parte, el factor edad resulta imprescindible para constatar en qué medida se presenta el cambio lingüístico. García (2015) realiza una importante acotación:

Los grupos generacionales disponen de un protagonismo más que significativo para aquilatar la aparición y propagación de las innovaciones lingüísticas. No es que todas ellas procedan única y exclusivamente de los hablantes de menor edad, pero sí que comparativamente es ahí, en 
los tramos generacionales de la adolescencia y la juventud, donde mayor actividad se consigna (p. 126).

Hernández (1991) concuerda con que el habla juvenil presenta una abundante creación léxica, agudeza y utilización de recursos de cambio léxico en la lengua, todo ello obedeciendo a la intención no solo comunicativa, sino también social: el deseo de «romper los moldes convencionales de la lengua estándar», y de brindarle una característica especial al grupo al que pertenece.

Tal deseo de pertenencia es importante para tener en cuenta los factores psicolingüísticos que condicionan la comunicación informal, amical o familiar. Estos factores mediatizan el lenguaje y permiten la inteligibilidad de los interlocutores. Consideraremos, pues, como un factor clave para el presente estudio el de la afectividad, propuesto por Hernández en 1991:

Afectividad muy acusada, que se manifiesta y/o resuelve en diversos rasgos, como son un automatismo en la expresión, una actitud psíquica más o menos relajada de los hablantes, espontaneidad comunicativa, improvisación, y la superposición y potenciación de códigos diferentes (verbal, cinésico...), etc. En este factor influye extraordinariamente el tipo de relación entre los hablantes, la situación psíquica de los mismos y la intención que lleva el mensaje (p. 12).

Así, podemos decir que los factores sociales, cuya alta interdependencia refleja la propia dinámica social en sentido amplio (García, 2015), se encontrarán condicionados por la actitud que tome el hablante durante la situación comunicativa, y solo la fusión de estos factores permitirá que se presente un cambio en el módulo lingüístico que dé cuenta de las necesidades de los hablantes cuando se encuentran en un grupo social determinado.

\subsection{El proceso de metátesis}

La metátesis es un proceso de innovación léxica que se utiliza en la creación de jergas. Una jerga o lenguaje especial es «un lenguaje de grupo 
no definido por criterios geográficos, culturales, ni temporales, sino por criterios socio-situacionales [...]» (Roffé, 1996: 201). Este mismo autor nos dice que dentro del vocablo jerga quedan incluidos los dialectos sociales, como el argot del hampa y el habla juvenil, y los registros situacionales como las jergas ocupacionales, técnicas, semi técnicas, científico-técnicas, las hablas sectoriales y los argots. Por otro lado, el término argot es usado, a veces, como sinónimo de jerga, pero para referirse a una lengua secreta, atribuida a maleantes, vagabundos o prostitutas, como medio de información codificado u oculto para terceros (Ramírez, 1996).

Dado que la concepción más extendida de argot es que es una lengua secreta o, por lo menos, usada por un grupo social muy específico que tiene como fin el secretismo de su uso, no calza con el uso extendido que se hace de las innovaciones léxicas por metátesis que aquí estudiaremos. Por lo tanto, si entendemos la jerga en un sentido más general, como lo propone Roffé (1996); es decir, como un uso particular, pero no solo hablado por personas del hampa, sino que también está presente en el habla juvenil ni que su función principal es el secretismo sino que involucra otros criterios situacionales. En tal sentido, obtenemos un término útil para los fines que busca este trabajo.

Ahora bien, la creación de jergas es parte de la innovación léxica de toda lengua. Aguirre y Molina (2013) señalan que este tipo de productividad léxica y semántica puede ser explicada atendiendo al fenómeno de motivación y los mecanismos de creación léxica, y nos advierten:

En principio, se debe aclarar que aunque el argot [o la jerga] engloba un léxico particular, su configuración está determinada por los mismos mecanismos que rigen el léxico de la lengua común, por tanto, apela a los procedimientos de esta; solo que en el proceso hay un mayor realce de la expresividad mediante la creación metafórica, metonímica y el préstamo y, por supuesto, se presenta una mayor conciencia sobre la motivación (p. 41).

De esta manera, es importante resaltar que la creación o innovación léxica mediante jergas por metátesis está motivada prominentemente 
por una necesidad expresiva, aunque también encontramos otros desencadenantes que pueden ser de tipo cognitivo como los tabúes y eufemismos, entre otros.

En cuanto a la clasificación de la metátesis - como forma de creación de jergas - con respecto a otro tipo de jergas, Quesada (1999) elabora una catalogación formal de lo que él llama «juego de palabras» o «juego lingüístico». Él toma como base los niveles o componentes de la estructura lingüística en los que actúa este juego lingüístico, a saber, fonético, morfológico y léxico. El autor incluye la metátesis o «inversión de segmentos y sílabas» (como él las denomina), dentro del nivel fonético, junto con otros como la alteración segmental, fonotáctica extranjera y rimas. Sin embargo, para Escobar y Hualde (2010) este proceso que involucra el cambio en el orden de las sílabas para la creación léxica de jergas es morfológico. Asimismo, Salillas (1896: 24-66) incluye el fenómeno de trasposición (lo que en este trabajo llamamos metátesis) dentro de uno más general que denomina «alteraciones fonéticas» y «formas de permutación» en los que describe algunos procedimientos argóticos de creación de palabras, basados sobre todo en «disimulos fonéticos, inversión de sílabas, cambios de vocales, añadido y mutación de desinencias, metátesis, transposiciones, supresiones y aumento de sílabas».

En este trabajo, proponemos la denominación de metátesis en el sentido de permutación del orden de las sílabas de una palabra que trae como resultado la creación de una jerga. Adicionalmente al proceso de metátesis, pueden ocurrir otros fenómenos como la eliminación de sílabas (apócope o síncopa), metátesis segmental, epéntesis o elisiónde segmentos, etc.; sin embargo, se reconoce que la metátesis es el procedimiento dominante para la innovación léxica.

\section{Metodología}

El presente artículo tiene un enfoque sociolingüístico. Su alcance se encuentra limitado a la población de los estudiantes de pregrado de la Universidad Nacional Mayor de San Marcos (UNMSM), distribuidos en cinco áreas académicas: Ciencias de la Salud (A), Ciencias Básicas (B), 
Ingenierías (C), Ciencias Económicas y de la Gestión (D) y Humanidades y Ciencias Jurídicas y Sociales (E). La muestra seleccionada consta de cuarenta estudiantes, ocho por cada área.

El estudio se realizó en los meses de mayo y junio del 2019. Para la empresa del mismo, se elaboró una encuesta con un total de veinte jergas generadas por metátesis. La técnica utilizada fue la encuesta personal y virtual. Los estudiantes que fueron encuestados debían colocar una definición y crear una oración que utilizarían con su grupo de amigos a partir de la palabra propuesta, y dejar en blanco aquellas que no conocieran. Para guiar al colaborador, se le explicó cómo resolver la encuesta y se le indicó que no debía ser ayudado por otra persona; ya que las respuestas debían ser personales para realizar adecuadamente el estudio. En este sentido, fueron mejor resueltas las encuestas enviadas por un medio virtual o las que fueron hechas a personas que se encontraban solas y no junto con un grupo.

\section{Análisis}

Las encuestas elaboradas para la presente investigación arrojaron diversos datos que nos permiten observar cómo se da el uso de las jergas creadas por procesos de metátesis entre los universitarios limeños. Los datos fueron obtenidos de estudiantes cuyo rango de edad varía entre los 18 y los 28 años; es decir, los encuestados fueron personas nacidas en la década de 1990 y los primeros años del 2000. Al respecto, sabemos que los colaboradores forman un grupo homogéneo que pertenece a una misma generación, y que todos ellos continúan en la etapa de la juventud y adultez temprana.

Las jergas pertenecientes al corpus realizado fueron divididas en dos grupos, como se muestra en la Tabla 1:

\begin{tabular}{|c|c|}
\hline \multicolumn{2}{|c|}{ Jergas generadas por metátesis } \\
\hline Sexuales & No sexuales \\
\hline chopin & choborra \\
\hline gapin & fercho \\
\hline
\end{tabular}

Bol. Acad. peru. leng. 66(66), 2019 / e-ISSN: 2708-2644 
https://doi.org/10.46744/bapl.201902.002

\begin{tabular}{|c|c|}
\hline jeropa & grone \\
\hline nepe & jerma \\
\hline nopor & jonca \\
\hline telo & lompa \\
\hline \multirow{4}{*}{} & lonsa \\
\cline { 2 - 2 } & merfi \\
\cline { 2 - 2 } & mica \\
\cline { 2 - 2 } & ñoba \\
\cline { 2 - 2 } & ponja \\
\cline { 2 - 2 } & rioba \\
\cline { 2 - 2 } & tolaca \\
\hline
\end{tabular}

Tabla 1. Jergas creadas por metátesis distribuidas entre las que refieren a un concepto sexual y las que no lo hacen.

Destacamos la palabra jerma, debido a que, si bien no siempre es utilizada en un contexto sexual, su uso es bastante afín al presentado en dicho grupo: jerma no es solo definida como 'mujer', sino preponderantemente como 'enamorada', 'novia', 'pareja'. Así lo demuestran dos ejemplos proporcionados por nuestros colaboradores. El primer aporte es de una colaboradora del género femenino.

(1) «La jerma se tiró a todos»

El que el término jerma es usado en el sentido de mujer, pero claramente en un contexto sexual. El segundo, por otro lado, nos lo brinda un colaborador del género masculino.

(2) «Ese pata no tiene jerma, es más virgen»

Este ejemplo también se presenta en un contexto sexual, pero en el que el término hace referencia a 'pareja', 'enamorada' o 'novia'. Esta relación es importante, debido a que son las jergas sexuales las más conocidas y utilizadas por los hablantes encuestados, en contraste con las jergas del grupo e índole no sexual. 
Podemos decir, como hipótesis inicial, que los grupos de jóvenes universitarios, en la actualidad, enfocan su creatividad lingüística a un tipo de creación léxica que les permita pertenecer a un grupo determinado, en el cual sean capaces de expresar la familiaridad y cercanía de los allí presentes en contextos donde puedan, en palabras de Hernández (1991), «improvisar».

Los resultados sociolingüísticos se expondrán, a continuación, de acuerdo con las variables de género, área académica y distrito de residencia, respectivamente.

\subsection{Variación por género}

En primer lugar, observamos en qué medida los estudiantes varones conocían las jergas propuestas en contraste con el conocimiento de las estudiantes mujeres. Así, obtuvimos el siguiente resultado:

\begin{tabular}{|l|c|c|}
\hline Género & Sexuales & No sexuales \\
\hline Masculino & 5,01 & 9,05 \\
\hline Femenino & 3,15 & 6,93 \\
\hline
\end{tabular}

Tabla 2. Promedios de la cantidad de jergas originadas por metátesis utilizadas por los estudiantes según su género, con base en un corpus de veinte palabras.

Los promedios fueron obtenidos con base en seis jergas sexuales y catorce no sexuales (como se aprecia en la Tabla 1). Respecto de ello, podemos observar que en ambos casos son los hombres quienes cuentan con un mayor conocimiento y utilización de las jergas, en una proporción similar en ambos grupos.

Un ejemplo que evidencia de manera clara lo antes mencionado es la mayor incidencia en el uso de gapin por los hombres, pues la recurrencia del uso de esta jerga es tres veces mayor en hombres que en mujeres. Consideramos que esto obedece a un conocimiento activo o pasivo que se tiene de gapin. Creemos que las mujeres tienen un conocimiento pasivo 
de esta jerga, pues, por lo general, solo la escuchan; pero no la utilizan, y cuando lo hacen se limitan a repetir lo que escuchan de los hombres, como en el siguiente ejemplo:

(3) «A chupetín Trujillo le gusta la gapin.»

Por el contrario, los hombres poseen un conocimiento activo que se expresa en su uso, sobre todo para hacer bromas pesadas o dar calificativos físicos o apodos, como vemos en los siguientes ejemplos:

(4) «El pelado cabeza de gapin»

(5) «Cara de gapin»

(6) «Pareces una gapin parada»

(7) «Derly me lame la gapin»

(8) «Me llegas al gapin»

Además, es importante acotar que algunos colaboradores usaban gampi en lugar de gapin, lo cual evidencia una alternancia entre estos dos términos.

Cabe recalcar, por último, que de acuerdo con cada área académica sí se presentaron variaciones sustanciales respecto del resultado general. En el área B, por ejemplo, el promedio de jergas sexuales y no sexuales utilizadas fue de 4 y 5,6 en hombres y de 2,33 y 5,67 en mujeres, respectivamente. Vemos, pues, que la variación empieza a ser mayor entre el conocimiento de jergas sexuales y de jergas no sexuales, siendo estas últimas utilizadas prácticamente en la misma medida por ambos géneros. Los resultados generales de ambas variables (género y área académica) serán presentados en el siguiente punto.

\subsection{Variación por área académica o profesión}

Respecto de las áreas académicas, obtuvimos como resultados generales los expuestos en la Tabla 3. 
https://doi.org/10.46744/bapl.201902.002

\begin{tabular}{|c|c|c|}
\hline Área & Sexuales & No sexuales \\
\hline A & 3,38 & 6,63 \\
\hline B & 3,38 & 5,63 \\
\hline C & 3,63 & 6,75 \\
\hline D & 4,5 & 9,13 \\
\hline E & 5,13 & 11,63 \\
\hline
\end{tabular}

Tabla 3. Promedios de la cantidad de jergas originadas por metátesis utilizadas por los estudiantes según su área académica, basados en un corpus de veinte palabras.

A partir de la Tabla 3, vemos de qué manera varía el uso y conocimiento de las jergas generadas por metátesis a partir del área académica de los encuestados y, por tanto, de su profesión. Así, se muestra que los estudiantes de Ciencias Médicas, Ingenierías y Ciencias Básicas (áreas A, B y C) son quienes menor conocimiento tienen de nuestro objeto de estudio, mientras que los estudiantes de Ciencias Económicas y Administrativas y Ciencias Humanas y Letras (áreas D y E) son quienes más las utilizan. Aun así, cabe recalcar que, en cuanto a las jergas sexuales, más de la mitad de estas han sido reconocidas en todas las áreas, mientras que en lo que respecta a las jergas no sexuales únicamente han sido reconocidas más de la mitad en las áreas $\mathrm{D}$ y E, lo cual es un indicador de que, efectivamente, los estudiantes conocen y utilizan en mayor medida las jergas de índole sexual.

El hecho de que, en general, los estudiantes conozcan en mayor medida las jergas de índole sexual, y dado que la muestra seleccionada pertenece a una población homogénea en cuanto a su rango de edad, demuestra que el uso de jergas sexuales cohesiona a los jóvenes, los identifica con su propio grupo social y generacional, y genera un sentido de pertenencia entre ellos. Muestra de ello es que la mayoría de los contextos sexuales ofrecidos por nuestros colaboradores se refieren a sus amigos, como se puede ver en (9) y (10): 
(9) «Todos mis amigos y sus gansos disfrutan del nopor» (colaborador del género femenino, del área «D»)

(10) «Pareces una gapin parada», refiriéndose a un amigo» (colaborador del sexo masculino, del área $« \mathrm{C} »)$

Esta tendencia se repite, ya sea expresando la familiaridad con el interlocutor de manera directa o de forma indirecta.

Teniendo en cuenta los resultados obtenidos en 4.1 y 4.2 , organizamos en una tabla los promedios generales de las jergas utilizadas de acuerdo con el género y el área académica, ya que a partir de ello obtuvimos interesantes descubrimientos:

\begin{tabular}{|l|r|r|r|r|r|}
\hline Género Área & \multicolumn{1}{c|}{ A } & \multicolumn{1}{c|}{ B } & \multicolumn{1}{c|}{ C } & \multicolumn{1}{c|}{ D } & \multicolumn{1}{c|}{ E } \\
\hline Masculino & 14,33 & 9,6 & 14,5 & 15,5 & 16,67 \\
\hline Femenino & 7,2 & 8 & 6,25 & 11,75 & 17 \\
\hline
\end{tabular}

Tabla 4. Promedios de la cantidad de jergas originadas por metátesis utilizadas por los estudiantes de las cinco áreas académicas, con base en un corpus de veinte palabras.

Como podemos observar, son los estudiantes del área B (Ciencias Básicas), quienes presentan un menor conocimiento de las jergas originadas por metátesis, en contraste con el área E, en la cual existe un conocimiento mayor (siendo este el único área en el que el género femenino conoce y utiliza más dichas jergas que el masculino). Además, vemos una variabilidad preponderante en las áreas A (Ciencias de la salud) y C (Ingenierías), ya que el conocimiento de las jergas de los hombres supera por más de la mitad al conocimiento de las mujeres.

Es importante dilucidar de qué manera los estudiantes de las distintas áreas de estudio muestran su desarrollo creativo-lingüístico y cómo esta varía de área en área. Podemos decir, pues, que el área $\mathrm{E}$ (Humanidades y Ciencias Jurídicas y Sociales) presenta un mayor conocimiento de tales jergas debido, quizá, a que los estudiantes encuestados de dicha área poseen mejores habilidades comunicativas (sociabilidad y espontaneidad), inherentes a sus carreras; mientras que 
el área B muestra el menor conocimiento por parte de ambos géneros por la razón contraria. Tal acercamiento resulta importante para lo que veremos más adelante, al tocar el tema del sentido de pertenencia (que adelantamos en párrafos anteriores). La creatividad lingüística con base en el cambio léxico se desarrollará en contextos sociales que impliquen la pertenencia a un grupo social determinado.

Además, los datos que refieren al género de los encuestados permiten elucidar la gran variación existente entre ambos a pesar de pertenecer a una misma área, la cual se muestra en las áreas A y C. En ellas, se observa cómo claramente son los hombres quienes conocen y utilizan con mayor naturalidad tales jergas, mientras que las mujeres tan solo conocen una cantidad reducida de las mismas.

\subsection{Variación societal}

Al tratar la variable del distrito de residencia nos encontramos con los resultados organizados en la Tabla 5. Es preciso decir que, por motivos de economía, se ha considerado para el análisis únicamente aquellos distritos que tengan más de un residente de entre los encuestados, o que cuente con los encuestados con mayor y menor conocimiento acerca de las jergas creadas mediante el proceso de metátesis.

\begin{tabular}{|l|c|}
\cline { 2 - 2 } \multicolumn{1}{c|}{} & Promedio de jergas utilizadas \\
\hline San Juan de Lurigancho & 10,4 \\
\hline Lima & 12,25 \\
\hline Callao & 14,33 \\
\hline Villa María del Triunfo & 7,77 \\
\hline
\end{tabular}

Tabla 5. Promedio de la cantidad de jergas que los encuestados conocen y/o utilizan.

Tal y como se observa, en el Callao se encuentra la mayor cantidad de uso de jergas creadas por metátesis; por otro lado, resulta importante destacar que en Villa María del Triunfo se ha presentado una cantidad 
claramente más reducida, que se aleja por la mitad de lo que sucede en el distrito previamente señalado. Cabe destacar, de igual manera, que los encuestados que mostraron un conocimiento total de las jergas presentadas pertenecían a los distritos de Lince y La Victoria.

Socialmente hablando, podemos decir que la presencia de jergas creadas por metátesis se presentó preponderantemente en el uso dado por los hombres, mientras que las mujeres desconocían o no sabían de qué manera contextualizar cada palabra presentada. El punto de la contextualización mediante ejemplos fue clave para reconocer si realmente el colaborador conocía el significado de la jerga presentada, pues al contar con una lista de veinte palabras que seguían un mismo patrón estructural, los encuestados podían deducir la definición de estas al «solo voltear sus sílabas». Esto, sin embargo, no siempre sucedía de tal manera; así, obtuvimos los siguientes ejemplos en los que los colaboradores intentaron reconocer una palabra invirtiendo las sílabas:

A la palabra mica, que significa camisa, la definieron como cima

(11) «Fui a la cima del cerro a visitar a mi amiga» (colaborador femenino).

La palabra ponjafue definida como Japón

(12) Quiero ir a «ponja» (hace referencia al país).

Sin embargo, se usa como gentilicio para los nacidos en Japón, o de manera genérica para denominar a los asiáticos, como lo evidencian nuestros colaboradores:

(13) «Tenía una amiguita ponja.» (usado como gentilicio)

(14) «El dueño del chifa es un ponja.» (hace referencia a cualquier asiático).

Por otra parte, la mayoría de los encuestados pertenecía a distintos distritos, por lo cual no fue posible realizar una generalización mayor de la obtenida. Aun así, es importante recordar que es en los distritos Callao, Lima y San Juan de Lurigancho donde se ha presentado la media de un mayor uso de estas jergas. El distrito de Lima resulta 
puntualmente interesante, pues las costumbres, los hechos culturales e incluso la creatividad en el ámbito lingüístico de la ciudad fueron iniciados (y desarrollados), básicamente, en el contexto del distrito más antiguo.

\subsection{El uso de jergas como símbolo de pertenencia}

Con base en los factores sociales que han condicionado la presencia y el conocimiento de las jergas creadas por metátesis en los colaboradores, es importante señalar cuáles fueron las jergas más y menos utilizadas, lo cual permitirá aclarar nuestras intuiciones previas al análisis realizado.

En primer lugar, la jerga más conocida por los colaboradores fue jerma 'mujer', con un total de 39 encuestados que indicaron saber de ella; seguida de ñoba 'baño' y telo 'hotel', con 38 respuestas afirmativas. A modo de contraste, la jerga menos conocida y, por tanto, utilizada por los colaboradores fue mica 'camisa', pues tan solo cuatro de ellos respondieron su significado convencional, mientras que los otros indicaron no conocerla o, en su defecto, contextualizaron la jerga brindándole un sentido distinto y alejándola de su propia red semántica: la utilización de dicha jerga como 'mica bezona' fue uno de los ejemplos más importantes tanto para demostrar el desconocimiento del hablante como para dar cuenta de su propia creatividad ante un estímulo desconocido.

Es importante, además, relacionar el conocimiento de estas jergas por parte de los hablantes con la generación a la cual pertenecen (como indicamos y ejemplificamos en el epígrafe 4.2). Como vimos en principio, todos los encuestados son nacidos en la década del noventa y primeros años del dos mil. Jergas utilizadas en las décadas del cuarenta y cincuenta (como lo es mica) han sido desfazadas, y el uso que se les da hoy en día es, ciertamente, mínimo por la comunidad de hablantes más jóvenes. Sin embargo, aún consideramos las palabras baño o telo, igualmente utilizadas en décadas anteriores, debido a que la convención ha sido más fuerte. Además, cabe señalar que los hablantes jóvenes suelen darle mayor importancia a la economía de recursos en la creación léxica y estructuración lingüística: es mucho 
más sencillo el cambio de baño a ñoba que el cambio de mica a camisa; pues en esta última no solo surge un proceso de metátesis, sino también una elisión silábica y cambio de orden entre las sílabas (lo cual nos permitiría dilucidar, históricamente, que se trata de una jerga más antigua que las otras).

Al respecto de ello, es importante agregar que las palabras generadas solo por un proceso de metátesis fueron reconocidas de forma más sencilla por los encuestados, sin embargo, parece ser que esto se dio debido a sus intuiciones y, además, a la economía de la estructuración lingüística por la cual se caracteriza el habla juvenil. Aun más importante fue la convención que tienen estas palabras dentro de la sociedad y del grupo al que pertenecen, lo cual se muestra en la palabra que fue reconocida por más encuestados, independientemente del área en el que se encontrasen y del género al cual perteneciesen: nos referimos a la palabra jerma, que contiene más de un proceso lingüístico. Sin embargo, sí resultó importante la presencia de más de un proceso para el cambio en las palabras en el uso actual de las jergas, como sucede con mica al ser la palabra menos utilizada entre los encuestados.

Una muestra de la vivacidad de la palabra jerma entre los jóvenes es la adquisición de un sentido polisémico, como se señaló anteriormente; así, puede significar, de manera genérica, 'mujer joven', o adoptar el sentido preferido entre los jóvenes, es decir, significar 'enamorada', como lo muestra la mayor parte de la data recogida y que ejemplificamos a continuación:

(15) «Hoy tengo plan con mi jerma.» (enamorada)

(16) «Mi jerma es celosa.» (enamorada)

(17) «Esa jerma está buena.» （mujer joven)

(18) «La jerma se tiró a todos.» （mujer joven)

Además, puede adoptar distinta connotación al agregar los sufijos diminutivos o aumentativos, que en castellano expresan más que solo cantidad, como la expresión «iqué tal jermón!» o la obtenida de nuestros colaboradores: 


\section{(19) «Pedro tiene nueva jermita.»}

Para finalizar este apartado, añadimos que es posible determinar el uso de las jergas creadas por metátesis atendiendo a relaciones de pertenencia, como lo hemos explicado a lo largo del trabajo: las jergas creadas por procesos de metátesis, en efecto, son utilizadas en un círculo social determinado. Empero, consideramos que, debido a la extensión del presente artículo, es importante dejar como complemento una investigación más profunda de criterios pragmáticos como cercanía, familiaridad, formalidad e informalidad, que intervienen en el uso o desuso de las variedades jergales en cuestión, con base en otro tipo de instrumento y metodología que nos permita acceder a dicha información, a saber, la propuesta de contextos y premisas que desencadenen una potencial conversación semiespontánea entre un grupo de hablantes. Descubriremos, de tal manera, que ciertos usos jergales (de los que nos encargamos) se presentan en casos no necesariamente de familiaridad, pero sí de extrema informalidad, como sería una discusión o una pelea callejera.

\section{Conclusiones}

Primera. La variación en el uso de las jergas generadas por metátesis se encuentra claramente condicionada por los factores sociales de los hablantes. Si bien la variable etaria genera homogeneidad al referirnos a los jóvenes universitarios, el área académica a la que pertenecen los estudiantes muestra las diferencias del conocimiento y uso jergal: los estudiantes del área E, quienes presentan una actitud más sociable y mejores habilidades comunicativas, que se traducen en mayor espontaneidad, cuentan con un mayor conocimiento de las jergas generadas por metátesis, mientras que quienes no tienen estas exigencias comunicativas en sus carreras (área B) tienen menor conocimiento de dicho tipo de jerga y, al ser sometidos a ellas, buscan conocerlas con base en intuiciones. Confirmamos, además, que el género es un factor importante durante la creación léxica en ámbitos de mayor cercanía y extrema informalidad: los hombres cuentan con un mayor conocimiento y uso de las jergas generadas por metátesis que las mujeres. Así, las 
variables sociales, en conjunto, son imprescindibles para el estudio de la variación léxica dentro de un grupo o comunidad determinada.

Segunda. Confirmamos que el uso de jergas cohesiona un determinado grupo social. No es casualidad que el grupo de entrevistados (entre 18 y 28 años) reconozca en mayor medida las jergas sexuales creadas por metátesis que las no sexuales creadas por el mismo proceso.Sucede de igual manera con los contextos ofrecidos por nuestros colaboradores, en los que las jergas de tipo sexual son usadas en un contexto amical, demostrando así que el uso de este tipo de jergas, si bien tienen mucha carga expresiva e innovadora, fortalece los lazos de pertenencia de quien las emite y su grupo social, que es quien las acepta. Asimismo, obedeciendo a este sentido de pertenencia que apuntamos es que se deja de usar otro tipo de jergas "pasadas de moda», pero, generadas también por metátesis, como es el caso de mica. En este caso, el uso de la palabra mica ya no identifica al hablante como parte de un grupo social joven, sino, posiblemente, con un grupo de personas mayores; por ende, no es útil para establecer lazos de pertenencia entre los jóvenes, y estos las dejan de reconocer y las terminan olvidando. 
https://doi.org/10.46744/bapl.201902.002

\section{BIBLIOGRAFÍA}

AGUIRRE, J. y MOLINA, J. (2013). «Análisis léxico-semántico del argot de las reclusas de la cárcel "El Buen Pastor" de Bogotá». Lenguaje, 41(1), 35-57.

ÁLVAREZ, J. (2009). Diccionario de peruanismos. El habla castellana del Perú (2. ${ }^{a}$ ed.). Lima, Perú: Universidad Alas Peruanas.

AREIZA, R., CISNEROS, M. y TABARES, L. (2012). Sociolingü̈́stica: enfoques pragmático y variacionista (2. ${ }^{\mathrm{a}}$ ed.). Bogotá: Ocoe Ediciones.

BENDEZÚ, G. (1977). Argot limeño o jerga criolla del Perú. Lima: Librería Importadora Editorial y Distribuidora Lima S.A.

CALVET, L. (1994). L'argot. París: PUF.

DE MIGUEL, E. (Ed.). (2008). Panorama de la lexicología. Barcelona: Ariel.

ESCOBAR, A., HUALDE, J. (2010). La estructura de las palabras: morfología. En Hualde, J. et al. Introducción a la lingüística bispánica (123-200). New York, United State of America: Cambridge University.

ETXEBARRIA, M. (2013). La variación lingüística: precisiones en torno a la noción en diversas teorías lingüísticas. Oihenart, 28, 207-239.

GARCÍA, F. (2015). Sociolingüística. Madrid: Editorial Síntesis.

GUTIERREZ, G. (2014). Cambios y transformaciones lingüisticas en la jerga como construcciones idiomáticas (Tesis doctoral). Universidad Nacional de Educación Enrique Guzmán y Valle, Lima, Perú. 
https://doi.org/10.46744/bapl.201902.002

HERNÁNDEZ, C. (1991). «El lenguaje coloquial juvenil». Centro Virtual Cervantes, (38-39), pp. 11-20.

MONTES, J. (1983). Motivación y creación léxica en el español de Colombia. Bogotá: Instituto Caro y Cuervo.

QUESADA, J. (1999). «Algunos juegos de palabras en español: muestra y análisis estructural». Lexis,XXII(1), 167-180.

RAMÍREZ, L. (1996). Estructura y funcionamiento del lenguaje (7. a ed.). Lima: Derrama Magisterial.

ROFFÉ, A. (1996). «Desarrollo de la conceptualización y designación jergal hispano-francesa». Enmiendas propuestas. Revista de Filología Francesa, 9 (1), 201-210.

SALILLAS, R. (1896). El delincuente español. El lenguaje. Estudio filológico, psicológico y sociológico, con dos vocabularios jergales. Madrid: Librería de Victoriano Suárez.

SORBET, P. (2014). «Análisis lingüístico del vesre porteño». Roczniki Humanistyczne, LXII(5), 123-134.

. (2016). «En torno al tratamiento lexicográfico de los vesreísmos». Itinerarios, (23), 141-153.

TALMY, L. (1985). Lexicalization patterns: semantic structure in lexical forms. En Language typology and Syntactic description III: Grammatical Categories AND THE LEXICON (ED. T. SHOPEN). CAMBRIDGE UNIVERSITYPRESS, 57-149.

VARELA, D. (2016). Un sistema peculiar de creación de palabras en español: descripción y análisis de la homonimia parasitaria (Tesis de doctorado). Universidad Autónoma de Madrid, España. 
WARDHAUGH, R. y FULLER, J. (2015). An introduction to sociolinguistics (7. a ed.). West Sussex: John Wiley \& sons, Inc.

\section{ANEXOS \\ Anexo 1 \\ ENCUESTA \\ METÁTESIS EN LA CREACIÓN DE JERGAS}

La presente encuesta no tiene mayor intención que la de recopilar datos del uso de las palabras presentadas; por lo tanto, no existe respuesta buena o mala ni mejor o peor. En ese sentido, las definiciones no tienen que ser demasiado elaboradas, y deben ser hechas, en lo posible, con sus propias palabras. Asimismo, para crear las oraciones se pide a los colaboradores que sean lo más espontáneos posible, y que no se abstengan del uso de otras jergas o usos cotidianos o ya que eso ayudará con la riqueza de este trabajo.

I. Características generales del colaborador

1. Facultad:

2. Edad:

3. Género: F / M

4. Distrito de residencia:

Define las palabras que se presentan a continuación y crea una oración con ellas que utilizarías con tu grupo de amigos. En caso de que la palabra no te sea familiar, deja el espacio en blanco.

\section{Choborra}


Claudia Giselle Nieto Salas y Juan Gerardo Paco Saavedra https://doi.org/10.46744/bapl.201902.002

Chopin

Fercho

\section{Gapin}

Grone

Jerma

Jeropa

Jonca

Lompa

Lonsa

Lleca

Merfi

Mica 


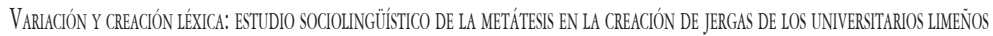
https://doi.org/10.46744/bapl.201902.002

Nepe

Nopor

$\overline{\tilde{N}} \circ b a$

Ponja

Rioba

$\overline{\text { Telo }}$

Tolaca

Si conoces otra palabra en la que se volteen las sílabas, similar a las presentadas previamente, escríbela, brinda su definición y un ejemplo. 


\section{Anexo 2}

\section{GLOSARIO}

En el presente glosario, exponemos la lista de palabras utilizada en nuestra encuesta acompañada de la que la origina. Únicamente ofreceremos una definición de las palabras que son menos familiares al lector, las que tomen dos o más significados en su uso o las que requieran alguna aclaración especial.

1. Choborra: 'borracho'

2. Chopin: 'pincho'

- Pincho. Sustantivo, masculino, vulgar. Pene.*

3. Fercho: 'chofer'

4. Gapin: 'pinga'

- Pinga. Sustantivo femenino coloquial. Eufemismo. Pene.*

5. Grone: 'negro'

- Grone. 1. Dicho de una persona de raza negra. Solo se usa en masculino.*

- Grone. 2. En la actualidad, también hace referencia a las personas que son hinchas del club deportivo de fútbol Alianza Lima.

6. Jerma: 'mujer'*

- jerma. En la actualidad, también hace referencia a enamorada.

7. Jeropa: 'pajero'

- Pajero. Dicho de una persona: Que masturba o se masturba.*

8. Jonca: 'cajón' o 'caja'

- Jonca. 1. Hace referencia al cajón peruano, un instrumento musical.

- Jonca. 2. En la actualidad es más común que se use para referirse a una caja de cervezas

9. Lleca: 'calle'

10. Lompa: 'pantalón'

11. Lonsa: 'salón'

12. Merfi: 'firme'

- Firme. 1. Dícese de algo que está muy bueno.* 
https://doi.org/10.46744/bapl.201902.002

- Firme. 2. Dícese de algo que es legal, de buena procedencia, un producto original, contrario a un producto pirata o de dudosa procedencia.

- Firme. 3. Dícese de un enunciado, historia o promesa que es verdadero.

- Merfi. Toma únicamente el significado de la tercera acepción de 'firme'

13. Mica: 'camisa'

14. Nepe: 'pene'

15. Nopor: 'porno'

16. Ñoba: 'baño'

17. Ponja: 'japonés'

18. Rioba: 'barrio'

19. Telo: 'hotel'

20. Tolaca: 'calato, ta'

- Calato, ta. 1. Desnudo, en cueros.*

- Calato, ta. 2. Dicho de un niño: Que está recién nacido.*

- Calato, ta. 3. Dicho de una persona: que está pobre.*

- Tolaca. Toma únicamente el significado de la primera acepción de 'calato'.

Nota: las acepciones que se encuentran marcadas con (*) son recogidas del Diccionario de Peruanismos, de Juan Álvarez Vita, consignado en la bibliografía. 